\title{
DISEÑO E IMPLEMENTACIÓN DE UN CONTROLADOR PROPORCIONAL INTEGRAL DERIVATIVO PID, EN UN ARREGLO ANALÓGICO PROGRAMABLE EN CAMPO FPAA
}

Por:

José Armando Becerra Vargas'. Manuel Alejandro Maldonado Rojas $^{2}$. Mayra Johanna Toscano Bustamante ${ }^{3}$

\section{RESUMEN}

En el presente artículo se describe el desarrollo del prototipo de un controlador PID, implementado en un arreglo analógico FPAA (Field Programmable Analog Array), diseñado y construido en el laboratorio de microelectrónica de la universidad Francisco de Paula Santander y el grupo de investigación en automatización y control (GIAC) y validado sobre un el control de velocidad para motor DC del módulo MRCC900 de ALECOP del laboratorio de Electrónica de Potencia del Servicio Nacional de Aprendizaje SENA, regional Norte de Santander.

PALABRAS CLAVE: Microelectrónica, PID, FPAA, Microcontroladores, motor DC, Anadigm.

\section{ABSTRACT}

The present article the development of the prototype of a controller PID is described, this is implemented in an analog array FPAA (Field Programmable Analog Array), designed and constructed in the laboratory of microelectronics of the Francisco de Paula Santander university and the group of investigation in automation and control (GIAC), validated with the control of speed for motor DC of the MRCC900 ALECOP module, installed in the Power Electronics laboratory of the Servicio Nacional de Aprendizaje SENA, regional Norte de Santander.

Keywords: Microelectronics, FPAA, PID, Microcontroller, DC motor, Anadigm.

\section{INTRODUCCIÓN}

Los FPAAs son el equivalente analógico de los FPGAs; estos dispositivos análogos configurables permiten el procesamiento analógico de señales, tales como filtrar, sumar, caracterizar, rectificar, generar y otras funciones. Se basa en la técnica del capacitor switcheado, que consiste en hacer una equivalencia de una red $\mathrm{RC}$ a través de un capacitor y la alternancia de un arreglo de switches [1]. Un FPAA esta conformado por bloques análogos configurables, una red de interconexiones programables y un registro para almacenar los bits de configuración; además posee una interfaz digital para recibir datos configuración de las diferentes topologías de los circuitos de las funciones analógicas.

Con el uso de FPAAs se simplifica el proceso de diseño analógico, se reduce el tiempo de implementación, y se habilita la reconfiguración en tiempo real de funciones analógicas dentro de un mismo sistema [2].

El FPAA utilizado es un AN220E04 de Anadigm ${ }^{\circledR}$, este dispositivo se escogió por la capacidad de 4 bloques análogos configurables, $2 \mathrm{MHz}$ de ancho de banda, reconfiguración dinámica, interfaz digital de configuración SPI, y su software de desarrollo ANADIGMDESIGNER2, ofrece la posibilidad de trasladar diseños de topologías de circuitos a código $C$; permitiendo que el diseño sea ajustado y controlado por microprocesadores dentro de un sistema embebido.

Aprovechando las soluciones que ofrecen los dispositivos análogos programables, se construyó un controlador PID con la capacidad de ajustar las constantes en tiempo real, que es comandado por un sistema digital independiente y autónomo; en

\footnotetext{
'Universidad Francisco de Paula Santander. Docente Tiempo completo, director del Grupo de Investigación en Automatización y Control "GIAC". Ingeniero Electricista, Especialista en Ingeniería Hospitalaria, Candidato a Magíster en Controles Industriales. jabecerr@bari.ufps.edu.co.

2Universidad Francisco de Paula Santander. Facultad de Ingeniería. Ingeniero Electrónico. maldonadorma@hotmail.com.

3Universidad Francisco de Paula Santander. Facultad de Ingeniería. Ingeniera Electrónica. maitos@gmail.com.
} 


\section{DISEÑO E IMPLEMENTACIÓN DE UN CONTROLADOR PROPORCIONAL INTEGRAL DERIVATIVO PID, EN UN ARREGLO ANALÓGICO PROGRAMABLE EN CAMPO FPAA}

el que se almacenan las funciones del circuito PID, configura el dispositivo analógico programable, maneja y procesa la información de la consola de programación y ejecuta el Auto-Set-Point; este último como complemento para las diferentes aplicaciones en que se desee emplear el controlador PID.

\section{MATERIALES Y MÉTODOS}

El desarrollo del controlador se realizó en las instalaciones del laboratorio de Electricidad y Electrónica y el Grupo de investigación en Automatización y control "GIAC", ubicados en la sede principal de la Universidad Francisco de Paula Santander.

El controlador consta de una consola de programación construida con una pantalla LCD 4×20 Alfanumérica y un teclado matricial de $4 \times 4$; una tarjeta de circuito impreso con un FPAA AN220E04, un microcontrolador Atmega 16 de la familia AVR, un circuito de conversión de señal digital-análoga basado en un DAC0800 y un bloque de amplificadores operacionales para el acondicionamiento de las señales analógicas con el FPAA.

Figura 1. Diagrama de Bloques del Controlador PID.

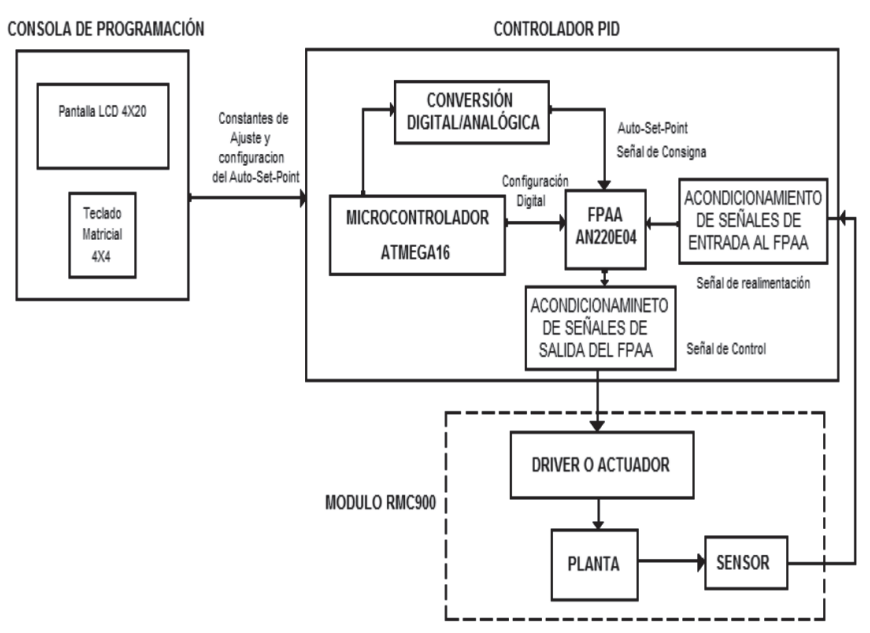

\section{CARACTERISTICAS DEL AN220E04}

Los FPAAs se basan en la tecnología de capacitor switcheado que implementa una resistencia equivalente por la alternancia de switches en las entradas de un capacitor, sus principales características es que un solo capacitor equivale a una red $R C$, el área que ocupa es menor que una resistencia, amplio rango de valores de resistencias, tolerancia típica de +/$1.0 \%$ y valores de RC altamente independientes de la temperatura [3].

Con base en la técnica de capacitor switcheado se forman los Bloques Análogos Configurables CABs. Los CABs constan de bancos de interruptores análogos - matriz de switches, que pueden ser estáticos para determinar la topología del circuito, los valores de resistencias y entradas activas, o dinámicos que manipulan la señal análoga de entrada; también constan de capacitores programables, amplificadores operacionales y un comparador; a demás cuenta con funciones adicionales coma la tabla de búsqueda LUT y contador programable, útiles para la generación de funciones no lineales. A continuación se muestra la arquitectura de un bloque análogo configurable [4].

Figura 2. Arquitectura de un bloque análogo configurable.

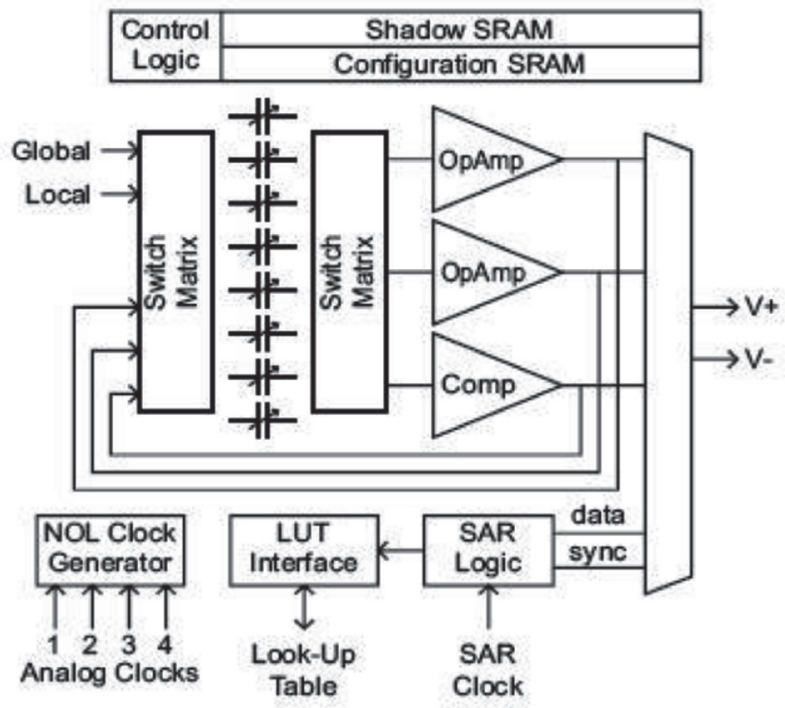




\section{DISEÑO E IMPLEMENTACIÓN DE UN CONTROLADOR PROPORCIONAL INTEGRAL DERIVATIVO PID, EN UN ARREGLO ANALÓGICO PROGRAMABLE EN CAMPO FPAA}

El FPAA AN220E04 de Anadigm ${ }^{\circledR}$ presenta reconfiguración dinámica, un ancho de banda típico $2 \mathrm{MHz}$, SNR entre 80 y $100 \mathrm{~dB}$, tres entradas y dos salidas dedicadas, una entrada multiplexada $4: 1$, una tabla de búsqueda LUT de 256 bits, THD de $80 \mathrm{~dB}$ y encapsulado de 44pines QFP(10x10×2mm).[5]

Figura 3. Arquitectura de un AN220E04.

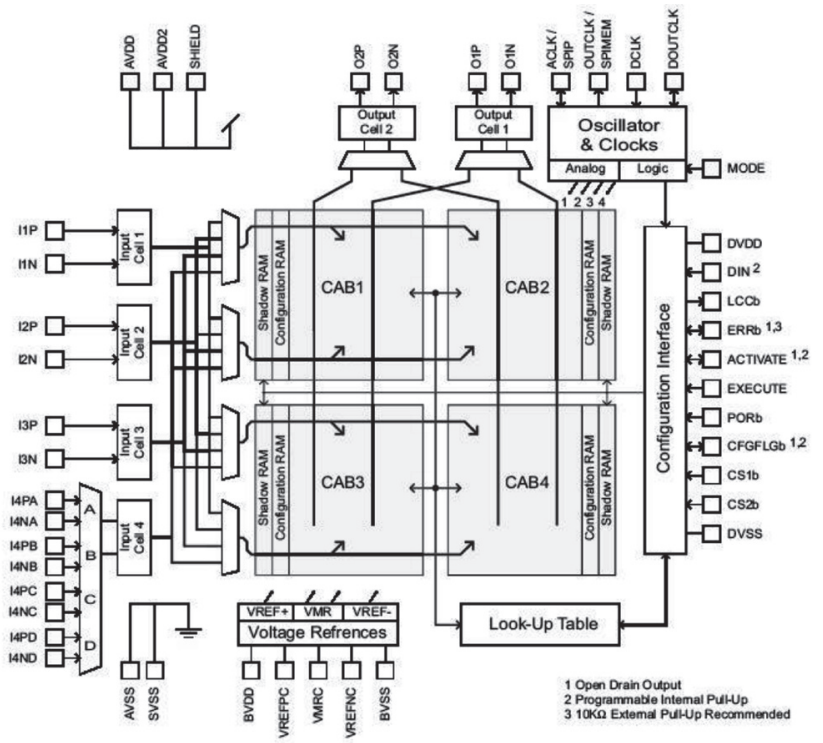

AnadigmDesigner2 es el software de Anadigm que soporta el diseño de circuitos analógicos en los AN220E04 [6,7], este software es de libre se descarga de la pagina de Internet de Anadigm y luego se pide la licencia ID al registrarse. El software permite procesar señales analógicas programables dinámicamente (dpASP), substituye fácilmente un circuito discreto alcanzando mayor exactitud, permite adaptar un circuito analógico a los cambios ambientales y a las condiciones de funcionamiento en tiempo real, reduce drásticamente el tiempo de diseño, gran exactitud en circuitos análogos, extensa documentación de ayuda, osciloscopio virtual incorporado, interfaz intuitiva para el usuario entre otros [8].

\section{MICROCONTROLADOR AVR}

El microcontrolador ATMegal6 de la familia AVR

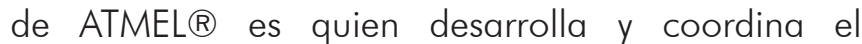

funcionamiento del controlador PID y cuenta con las siguientes características, arquitectura RISC, código totalmente transportable, memoria flash de $16 \mathrm{~Kb}$, memoria SRAM de $1 \mathrm{~KB}, 512$ bytes memoria EEPROM, logra un desempeño de hasta $16 \mathrm{MIPS}$ a $16 \mathrm{MHz}$, es decir una instrucción en un solo ciclo de reloi [9]. Para el desarrollo del proyecto se utilizaron las interfaces de comunicación SPI y UART; la programación se desarrolló utilizando la programación en sitio (ISP) y/ o el Boot-loader-Sopport con el software AVRDUDE y MEGALOAD respectivamente. La compilación se realizó con el compilador gratuito AVR-GCC de WINAVR [10].

\section{DISEÑO DEL CONTROLADOR PID}

Utilizando el software AnadigmDesigner2 se diseñó el control PID; aunque AnadigmDesigner2 trae un tools para control PID [11], este no se implemento debido a que la manipulación de las variables de ajuste es un tanto complicado y tediosa; además existen muchas formas de realizar el algoritmo PID, por lo que se utilizaron diferentes topologías circuitales hasta obtener finalmente una arquitectura sencilla que permitiera manejar directamente los valores de ajuste y realice correctamente la compensación de la señal de control[12].

Para el diseño de la topología circuital se insertan los CAMs (módulos análogos configurables) se ubican correctamente y se interconexionan; luego se despliega la documentación y se configura cada CAM seleccionando los parámetros de funcionamiento y el rango de los valores de ajuste.

Una vez obtenido el diseño de la topología circuital del controlador se procedió a generar las funciones en código $C$, para esto se utiliza el método de reconfiguración dinámica algorítmica, este método permite seleccionar las funciones en $C$ que varían los parámetros de los CAMs, como ganancia, tiempo de integración y tiempo de derivación, sin que cambie la arquitectura del circuito. 


\section{DISEÑO E IMPLEMENTACIÓN DE UN CONTROLADOR PROPORCIONAL INTEGRAL DERIVATIVO PID, EN UN ARREGLO ANALÓGICO PROGRAMABLE EN CAMPO FPAA}

Figura 4. Diseño del controlador PID en AnadigmDesigner2.

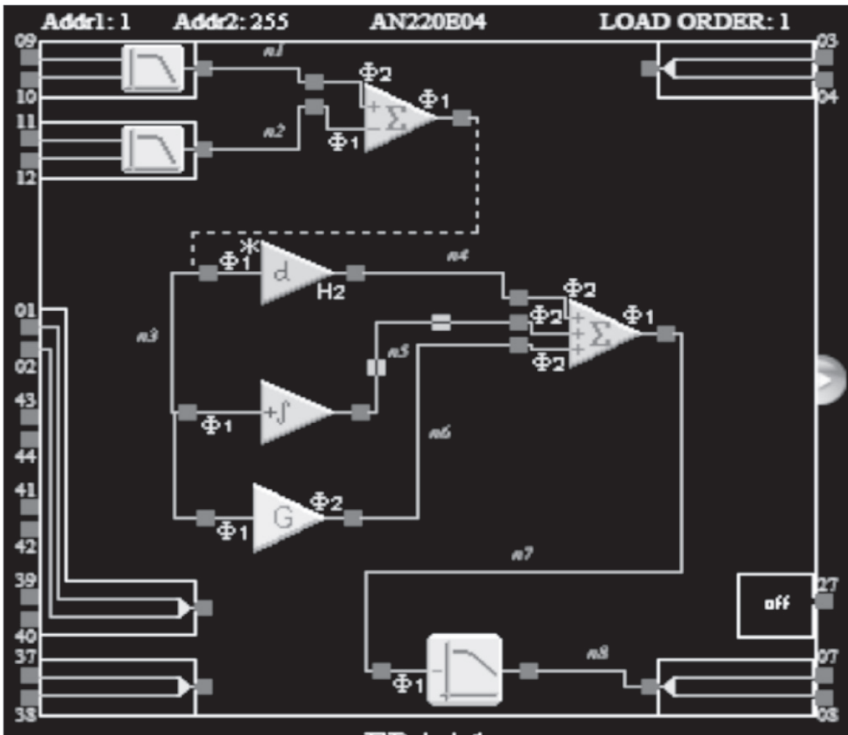

FPAA1

Logrado generar las funciones $C$ para la variación de los parámetros de las CAMs, se incluyen como librerías al compilador AVR-GCC de WINAVR [13], en el cual se desarrolla el programa para la ejecución del proyecto; se utilizó como apoyo para síntesis del código la comunicación con el Hyperterminal de Windows [14].

El desarrollo del código comenzó creando las funciones de generación de datos de configuración primaria y reconfiguración dinámica, y la descarga de datos utilizando el protocolo SPI al FPAA AN220E04; después se implementaron funciones para la variación de las parámetros de los CAMs, luego se creo una interfaz a través del Hyperterminal de Windows para ingresar los valores de ajuste de las constantes [15].

Una vez obtenidas las funciones del manejo del FPAA, se diseño el código del Auto-Set-Point, que genera señales de voltaje en periodos de tiempo específicos como señal de referencia para el controlador, y la conversión de la señal digital que genera el microcontrolador en una señal analógica a través de un circuito con un DAC0800; finalmente se diseño la interfaz de usuario mediante una consola de programación que despliega mensajes en la pantalla LCD 4×20 [16] y captura de información del teclado matricial $4 \times 4$; y la estructura del funcionamiento del Controlador PID con un Auto-Set-Point programable.

El funcionamiento del controlador es sencillo y práctico, la consola muestra una señal de bienvenida y luego despliega un menú con las opciones de Ajuste de Constantes del controlador, configuración del Auto-Set-Point y puesta en marcha del Auto-Set-Point; la opción de ajuste de constantes muestra los valores actuales de las constantes permitiendo escribir sobre ellas y una vez se actualicen el sistema descarga estos valores al FPAA tomando este ajuste en tiempo real.

Figura 5. Diagrama de funcionamiento del sistema.

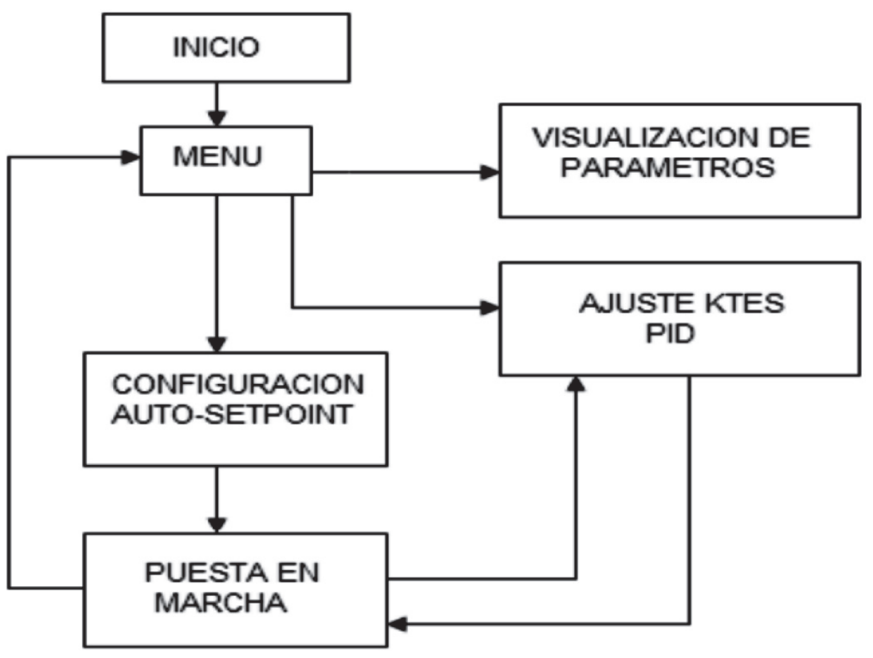

La opción de Configuración de Auto-Set-Point muestra los valores de la última configuración efectuada y pide el número de periodos a manejar y luego despliega uno a uno cada periodo para introducir el porcentaje de magnitud que se desea y el tiempo requerido para este incremento, decremento o periodo mantenido; a continuación se muestra una grafica de un ejemplo de configuración del Auto-Set-Point. 
DISEÑO E IMPLEMENTACIÓN DE UN CONTROLADOR PROPORCIONAL INTEGRAL DERIVATIVO PID, EN UN ARREGLO ANALÓGICO PROGRAMABLE EN CAMPO FPAA

Figura 6. Ejemplo de configuración del Auto-SetPoint.

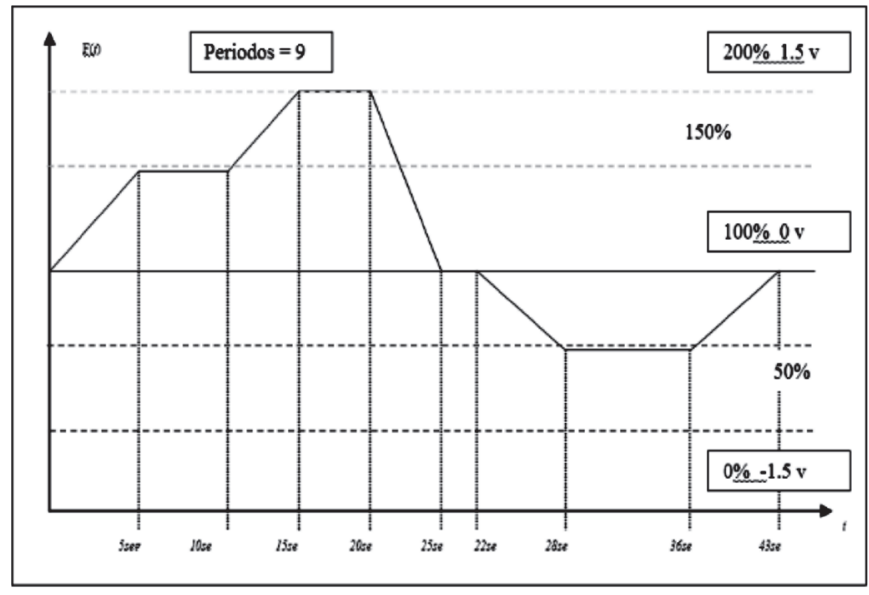

Uno de los aspectos más importante del desarrollo de aplicaciones con arreglos Analógicos Programables es la interfaz de las señales analógicas con el FPAA, debido a que estos dispositivos manejan diferentes configuraciones en su entrada como impedancias de entradas, filtraje, voltajes en modo común de referencia, excursiones de voltaje en modo diferencial [17].

Para el controlador se desarrollo la interfaz con las siguientes características: la interfaz de señales analógicas al FPAA convierte señales de entrada y/o salida en el rango de $-10 v a+10 v$ en modo simple a señales en modo diferencial en el rango de $0.5 \mathrm{v}$ a 3. $5 v$ con $2 v$ de nivel dc tomadas de la señal VMRC del FPAA como referencia $[18,19]$.

Figura 7. Acople de la señal de entrada al FPAA AN220E04

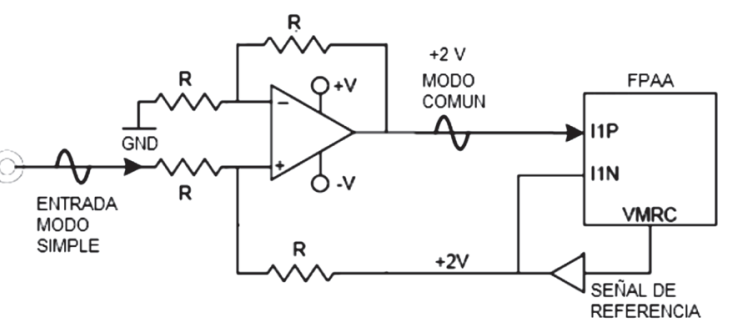

Figura 8. Salida del AN220E04 al módulo

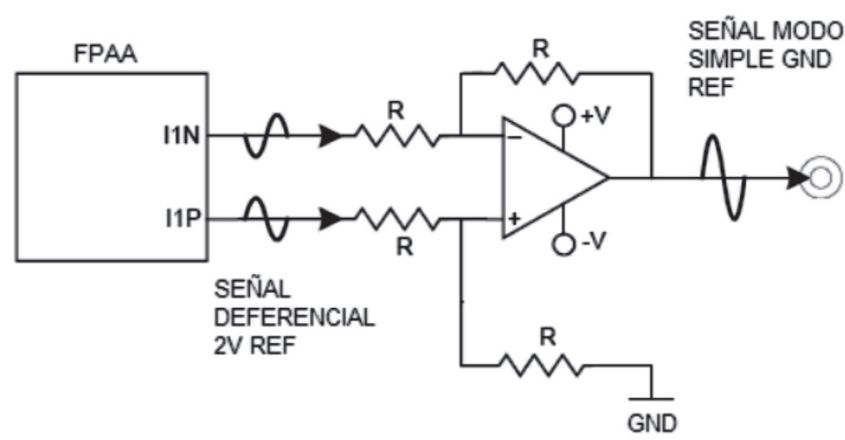

Una vez obtenida la estructura final del controlador PID, se procede al diseño del circuito impreso (PCB). Se utiliza el software EAGLE 4.11 $®$ (software CAD para el desarrollo de esquemáticos y circuitos impresos), partiendo desde el esquemático del proyecto hasta el diseño de la Board y generación de los archivos para la fabricación [20].

Figura 9. Diseño PCB del Impreso visto por las dos caras.

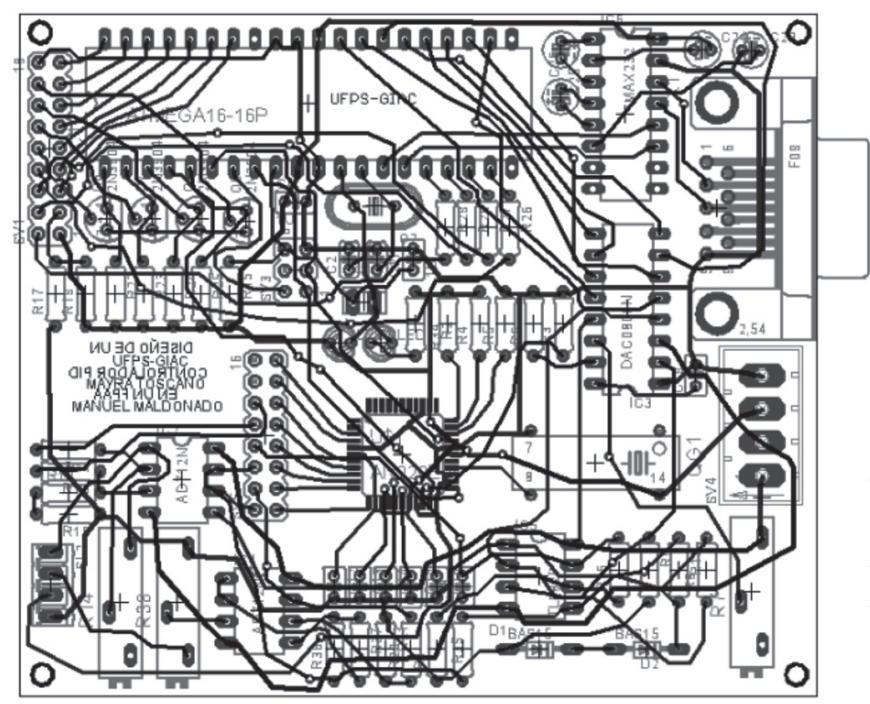

La construcción final de la tarjeta impresa es realizada por la empresa Microcircuitos LTDA de la ciudad de Cali.

Finalmente se realiza la prueba del controlador con la 


\section{DISEÑO E IMPLEMENTACIÓN DE UN CONTROLADOR PROPORCIONAL INTEGRAL DERIVATIVO PID, EN UN ARREGLO ANALÓGICO PROGRAMABLE EN CAMPO FPAA}

colaboración del laboratorio de Potencia del Servicio Nacional de Aprendizaje SENA, regional Norte de Santander, donde se hizo el ensamble y ajuste final del controlador PID. Para la validación del controlador se realizaron varias prácticas de control de velocidad de motores de C.C [21].

En la figura 10., se muestra una fotografía donde aparece; en la parte derecha, el módulo de regulación de velocidad MRCC900, en la parte central la tarjeta del controlador y su consola de programación, en la parte superior el motor de C.C., con el que se realizaron las prácticas y en la parte izquierda los instrumentos de medida.

Figura 10. Prueba de validación del controlador PIDFPAA.

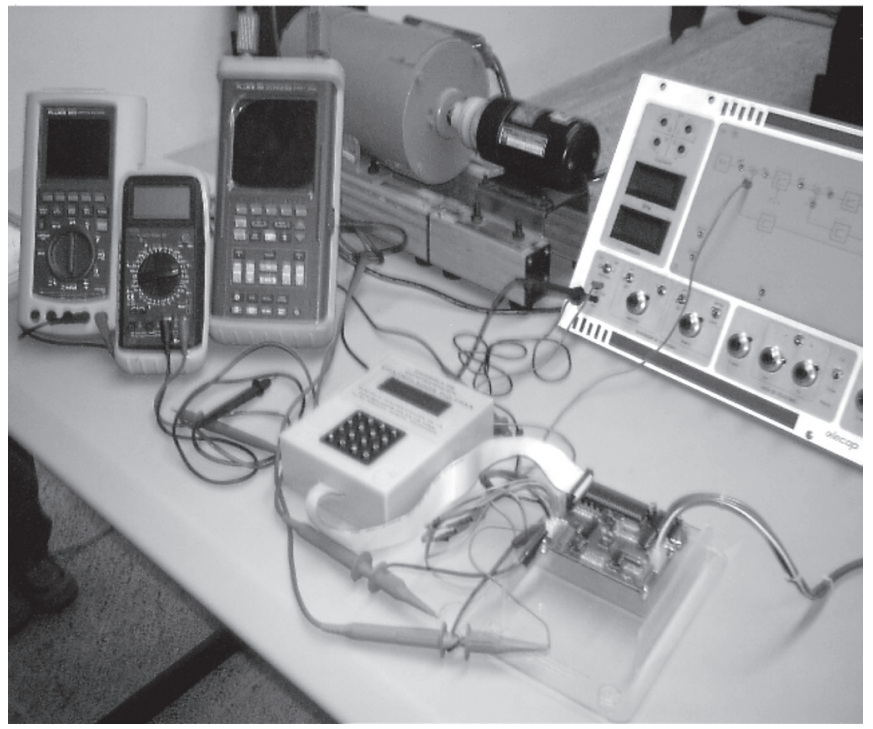

*Fuente: Autores.

\section{RESULTADOS}

Se desarrolla un controlador PID totalmente implementado en un FPAA, el cual ofrece un alto grado de fiabilidad en aplicaciones industriales, fácil manejo y flexible a la hora de hacer los ajustes, versátil para realizar configuraciones adecuadas a través del Auto-Set-Point y aplicable a cualquier sistema de control de lazo cerrado que requiera un dispositivo de regulación automática.

Las pruebas de validación del controlador fueron referidas al control de velocidad de un motor de corriente directa incluido en el módulo MRCC900 de ALECOP, del laboratorio de Electrónica de Potencia del SENA, regional Norte de Santander.

Se logra dar un paso muy importante en el desarrollo de proyectos de investigación en el área de la microelectrónica, el prototipo presentado en este artículo permite mostrar un desarrollo tecnológico de alto nivel en el área mencionada.

Este tipo de investigación permite reforzar y estimular la investigación y el desarrollo tecnológico en el Grupo de Investigación en Automatización y Control (GIAC), toda vez que el prototipo desarrollado en este proyecto ha arrojado excelentes resultados.

Se establece un vínculo de apoyo y cooperación con el Grupo de Microelectrónica de la Universidad Nacional de Colombia (GMUN) y el Servicio Nacional de Aprendizaje SENA, regional Norte de Santander.

\section{CONCLUSIONES}

Los FPAAs de Anadigm permiten el tratamiento de señales analógicas, trasladando circuitos complejos a un set de funciones simples por medio del software AnadigmDesigner2, facilitando así el tedioso y engorroso desarrollo manual de los diseños analógicos, ofreciendo capacidades de reconfiguración en tiempo real mediante una interfaz digital.

La línea de microcontroladores AVR de Atmel utilizada en el desarrollo del proyecto posee características que permitieron el manejo de periféricos, la programación del FPAA y el control del módulo; ofreciendo confiabilidad y versatilidad en las aplicaciones, debido a su alta inmunidad al ruido, facilidad en la programación ISP (In System Program) y amplias 


\section{DISEÑO E IMPLEMENTACIÓN DE UN CONTROLADOR PROPORCIONAL INTEGRAL DERIVATIVO PID, EN UN ARREGLO ANALÓGICO PROGRAMABLE EN CAMPO FPAA}

herramientas de diseño e información disponibles para el diseñador.

El lenguaje C utilizado para la programación del microcontrolador ATmegal6 permitió la inclusión de librerías de AnadigmDesigner2 y el manejo de las funciones generadas por cada CAM del FPAA, estas funciones fueron utilizadas para la Configuración Primaria y Reconfiguración Algorítmica.

Se diseñó e implementó un sistema análogodigital reconfigurable en tiempo real, totalmente independiente y autosuficiente, que permite manipular señales analógicas de control basadas en funciones del FPAA las cuales conforman el algoritmo de control PID; éste es reconfigurable digitalmente a través del microcontrolador ATmega 16.

Los sistemas implementados en arreglos analógicos programables, requieren tiempos muy cortos de desarrollo, además brindan la flexibilidad para el diseñador que no es posible encontrar con los circuitos analógicos tradicionales (cableados). Por tanto un sistema de esta naturaleza puede ser capaz de presentar varios tipos de comportamiento sin cambio alguno en su hardware.

El uso de un software y sus respectivas herramientas dispuestos para la creación de circuitos impresos PCB es de gran utilidad para la puesta en marcha del proyecto pues se logra una mejor presentación y un mejor funcionamiento del circuito.

\section{RECOMENDACIONES}

Implementar, dentro de la asignatura de Microcontroladores la documentación y uso de la familia ATmega, puesto que son los elementos que están revolucionando el uso de los microcontroladores en labores de control.

Es conveniente que mediante un proyecto de grado u otro mecanismo se ponga en marcha un laboratorio para el desarrollo de circuitos impresos, ya que con la miniaturización de los componentes en los proyectos la elaboración de los circuitos impresos es un tanto complicada de realizar mediante métodos artesanales.

Continuar con el desarrollo de proyectos de investigación en el área de la microelectrónica enfocados al uso de la tecnología de los FPAAs.

Estudiar la posibilidad de adquirir los Kits de desarrollo de FPAAs, para los laboratorios de Electricidad y Electrónica de la UFPS, y así los estudiantes puedan realizar aplicaciones reales con AnadigmDesigner2.

\section{BIBLIOGRAFIA}

[1] VINCENT GAUDET.FIELD - Programmable Analog Array FAQ [online]. University of Alberta, 2001.

http://www.ece.valberta.ca/ vgaudet/fpaa/faq.html. [Citado en 2004]

[2] Anadigm, Inc. Anadigmvortex Overview, PR021 100-0023, 2002.

http://www.anadigm.com/_doc/VXFPAAOV.pdf.

[Citado en 2005]

[3] Anadigm, Inc. Switched Capacitor. TheoryBasic SC and Anadigm- 1, 2002.

http://www.anadigm.com/_doc/BasicSC-tech.pdf.

[Citado en 2004]

[4] Anadigm, Inc. AN220e04 Overview, GF020800-0004, 2002.

http://www.anadigm.com/_doc/GF02080004.pdf. [Citado en 2004]

[5] Anadigm, Inc. Datasheet AN220E04, DS020700-U001e, 2002.

http://www.anadigm.com/_doc/DS020700-U001. pdf. [Citado en 2004] 


\section{DISEÑO E IMPLEMENTACIÓN DE UN CONTROLADOR PROPORCIONAL INTEGRAL DERIVATIVO PID, EN UN ARREGLO ANALÓGICO PROGRAMABLE EN CAMPO FPAA}

[6] Anadigm, Inc. AN121/220E04 User Manual, UM021200-U007e, 2002.

http://www.anadigm.com/_doc/UM021200-U007. pdf. [Citado en 2004]

[7] Ayuda de AnadigmDesigner2. [Viene con el software]

[8] Anadigm, Inc. AnadigmVortex,PR0309000038, 2003.

http://www.anadigm.com/_doc/PR080200-0001. pdf. [Citado en 2005]

[9] Atmel,Inc. ATMegal6 Datasheet, 2466J-AVR, 2004

http://www.atmel.com/dyn/resources/prod documents/doc2466.pdf. [Citado en 2005]

[10] WinAVR AVR-GCC. Para Windows.

http://winavr.sourceforge.net/.[Citado 2005]

[11] Tools AnadigmPID.

[12] Anadigm, Inc., PID Control Customer Presentation, PR080200-0004, Agosto 2002.

http://www.anadigm.com/_doc/FPAA Solutions for PID ControlPID Control.pdf. [Citado en 2005]

[13] Savannah.org. Manual de usuario del avrlibc.

http://savannah.nongnu.org/download/avr- libc/. [Citado en 2005]

[14] Ayuda de Hyperterminal de Windows.

[15] Anadigm, Inc., Dynamic Programming Starter Guide - Subwoofer Filter, Doc No. SK01SUBWU001.

http://www.anadigm.com/_doc/Dynamich\%20Progra ming\%20Guide\%20-\%20Subwoofer\%20Filter.pdf.

[Citado 2005]

[16] Peter Fleury`s Homepage http://homepage.sunrise.ch/mysunrise/peterfleury/ index.html. [Citado en 2005]

[17] Anadigm.Inc, Using the Anadigm ${ }^{\circledR}$ FPAA to Interface with Sensors - Technical Considerations. PR030600-0033, 2002

http://www.anadigm.com/_doc/ PR030600-0033. pdf. [Citado en 2005]

[18] Anadig.Inc, Anadigmvortex AN220D04 Evaluation Board User Manual, UM020800-U003s. 2004.

http://www.anadigm.com/_doc/ UM020800-U003s. pdf. [Citado en 2005]

[19] Anadig.Inc, AN221K04 Anadigmvortex Development Board User Manual, UM030900 U010e, 2003

http://www.anadigm.com/ doc/UM030900-U010e .pdf. [Citado en 2005]

[20] Cadsoft.Inc

http://www.cadsosft.de/. [Citado en 2005]

[21] ALECOP, Manual de Practicas del RMCC 900, Regulación de Velocidad de Máquinas de C.C. Versión 1.0 Septiempre 1998.

Fecha de recibido: Febrero 12 de 2007

Fecha de aceptación: Noviembre 23 de 2007 